\title{
Fast polymeric functionalization approach for the covalent coating of $\mathrm{MoS}_{2}$ layers.
}

Iván Gómez-Muñoz, ${ }^{1}$ Sofiane Laghouati, ${ }^{1}$ Ramón Torres-Cavanillas, ${ }^{1}$ Marc Morant-Giner, ${ }^{1}$ Alicia Forment-Aliaga, ${ }^{1}$ Mónica Giménez-Marqués ${ }^{1, *}$.

${ }^{1}$ Instituto de Ciencia Molecular (ICMol), Universidad de Valencia, c/ Catedrático José Beltrán 2, Paterna, 46980, Spain.

E-mail: monica.gimenez-marques@uv.es

Keywords: 2D materials; transition metal dichalcogenides; covalent functionalization; diazonium chemistry

We present the covalent coating of chemically exfoliated molybdenum disulfide $\left(\mathrm{MoS}_{2}\right)$ based on the polymerization of functional acryl molecules. The method relies on the efficient diazonium anchoring reaction to provoke the in situ radical polymerization and covalent adhesion of functional coatings. In particular, we successfully implement hydrophobicity on the exfoliated $\mathrm{MoS}_{2}$ in a direct, fast, and quantitative synthetic approach. The covalent functionalization is proved by multiple techniques including $X$-ray photoelectron spectroscopy and TGA-MS. This approach represents a simple and general protocol to reach dense and homogeneous functional coatings on 2D materials.

\section{Introduction}

Transition metal dichalcogenides (TMDCs) represent one of the most studied families of lamellar compounds that can be easily exfoliated into two-dimensional (2D) layers, exhibiting a plethora of unique physical and chemical properties. ${ }^{1}$ These 2D materials display an $\mathrm{MX}_{2}$ stoichiometry (where $\mathrm{M}$ is a transition metal and $\mathrm{X}$ is sulfur, selenium, or tellurium) and, depending on their structural arrangement, distinct polytypes with completely different catalytic, magnetic or electronic properties can be obtained. ${ }^{2-6}$ These physical features make TMDCs very attractive for their integration in 2D-based nanotechnologies such as optoelectronics or sensing. ${ }^{7,8}$ In this context, $\mathrm{MoS}_{2}$ is undoubtedly the flagship of the TMDCs family, due to its scalable preparation through simple exfoliation methods and amenable functionalization through chemical design. ${ }^{9,10}$

The molecular functionalization of $\mathrm{MoS}_{2}$ has been extensively explored to induce changes in its physical and mechanical properties, ${ }^{11}, 12$ modify its processability, or even add new functionalities. ${ }^{13-15}$ In this sense, different methods mainly involving electrostatic and/or covalent functionalization have been used to randomly distribute molecules on the 
surface, ${ }^{11,13,16-20}$ the former being generally preferred to ensure the chemical robustness of the final 2D functionalized system. Among covalent strategies, the chemistry of aryl radicals has been largely explored, even though a limited density of attached molecules is often attained (between $\approx 4-11 \%$ coverage) which correlates with a relatively inert surface. ${ }^{17,21}$ Therefore, the increase of functional groups on the $2 \mathrm{D}$ interphase is desired to efficiently modify its properties or to endow new functionalities provided by the anchored molecules.

This limited density issue has been recently faced by adding additional reducing agents such as $\mathrm{Kl}$, which promotes the massive reduction of aryl diazonium salts in solution leading to practically overall surface coverage. ${ }^{22} \mathrm{~A}$ suitable alternative is the use of reactive molecules capable of promoting in situ polymerization on the 2D surface. Several examples, including polymerization on graphene through imine-based chemistry ${ }^{23}$ and more recently in $\mathrm{MoS}_{2}$ using maleimides ${ }^{16}$ and poly(N-vinylcarbazole) have been reported ${ }^{24}$ These polymeric approaches ensure the presence of a dense coating in the final material, thus resolving the typically low degree of functionalization occurring in inert surfaces.

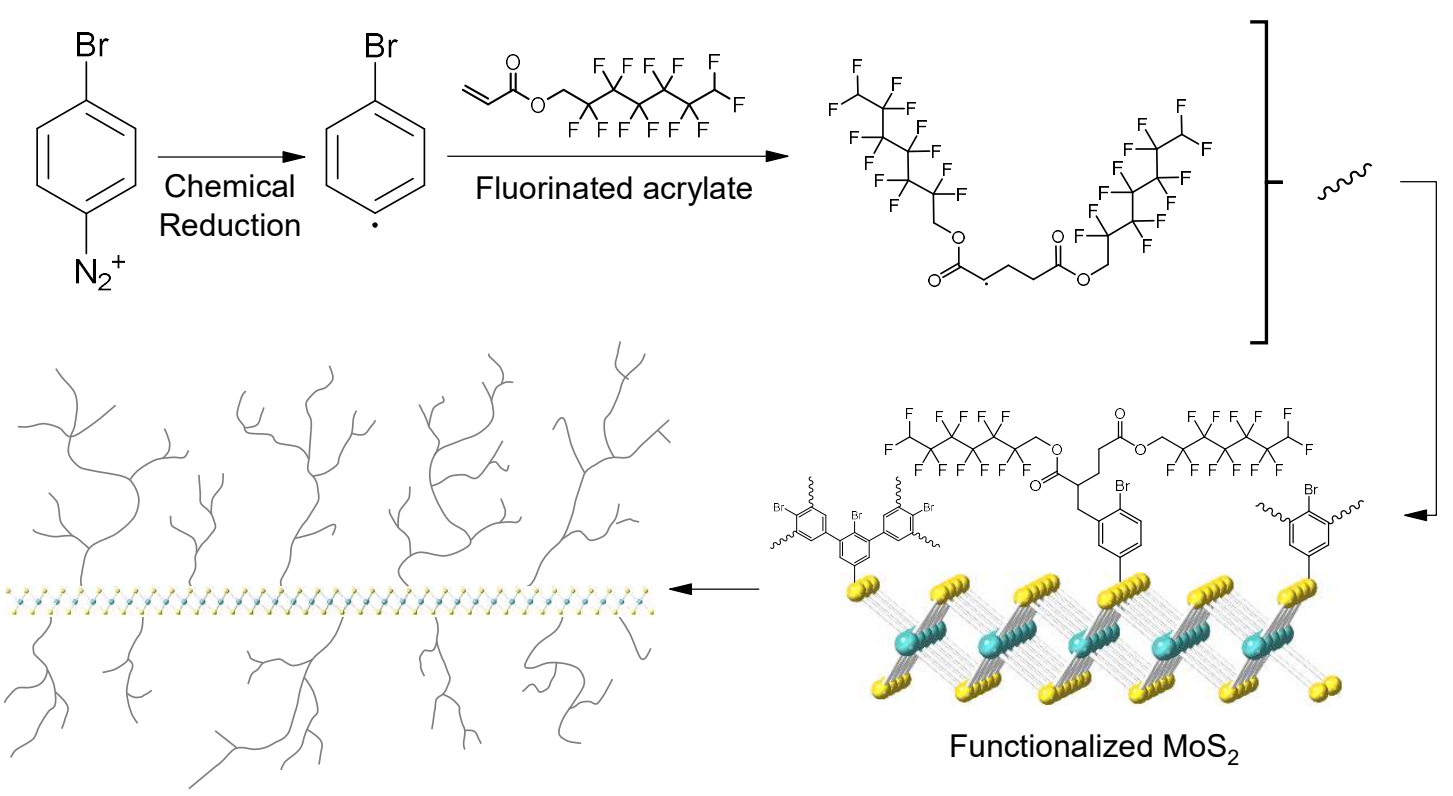

Figure 1. Schematic representation of the proposed polymeric reaction on $\mathrm{MoS}_{2}$.

Herein, we present a designed polymeric reaction to selectively coat chemically exfoliated (CE) $\mathrm{MoS}_{2}$ with a functional shell. We take advantage of the robust covalent archetypal diazonium grafting occurring via aryl radicals, combined with the polymerization of vinylic monomers. As a result, a large degree of functional moieties covering the $\mathrm{MoS}_{2}$ surface (Figure 1). ${ }^{25}$ The synthesis is adapted from a grafting method ${ }^{25}$ applied to a large number of materials with distinct nature 
such as carbon nanotubes ${ }^{26}$ and fibers, ${ }^{27}$ metal surfaces or metal oxide nanoparticles ${ }^{28}$ and recently to porous Metal-Organic Frameworks, ${ }^{29,30}$ but to the best of our knowledge, has not been used in TMDCs. This covalent surface reaction is particularly convenient since it occurs under fast mild conditions in a straightforward manner, leading to the anchoring of large amounts of molecules with the desired functionality. In this work, we selected the in situ formation of hydrophobic coatings, which serve as the basis to develop air-stable and more processable functional polymer-coated 2D materials.

\section{Results and Discussion}

Covalent adhesion of functional polyvinyl coatings onto $\mathrm{MoS}_{2}$. The quantitative grafting of functional polymers using a diazonium anchoring process was first adapted to functionalize $\mathrm{MoS}_{2}$ flakes (see Figure 1). The grafting process consisted of directly mixing a suspension of $\mathrm{MoS}_{2}$ flakes with an aryl diazonium salt in the presence of acrylate monomers. Two fluorinated acrylate monomers of different chain lengths were explored in parallel reactions, namely 1,1,1,3,3,3-hexafluoroisopropyl acrylate (acryl- $\left.\mathrm{C}_{3} \mathrm{~F}_{6}\right)$ and 2,2,3,3,4,4,5,5,6,6,7,7dodecafluoroheptyl acrylate (acryl- $\mathrm{C}_{7} \mathrm{~F}_{12}$ ). An aqueous colloid of CE $1 T-\mathrm{MoS}_{2}$ was first obtained following a previously reported method ${ }^{12}$ (see experimental section for further details). Two exfoliated suspensions were mixed in parallel reactions with an acetonitrile solution containing the aryl diazonium salt in a 1:3 molar ratio ( $\mathrm{MoS}_{2}$ :bromobenzene diazonium). Then, an excess of the corresponding fluorinated acrylate molecules (10 equivalents $\mathrm{MoS}_{2}$-based) were immediately added to the mixtures under vigorous stirring in air. The reactions were instantaneously initiated upon the addition of reagents and completed within a few seconds, as deduced by the fast formation of a black flocculate (see Figure S4). Essentially, the reaction is initiated by the well-known formation of aryl radicals that directly graft to the $\mathrm{MoS}_{2}$ surface forming a phenylene layer, as described previously. ${ }^{31}$ Simultaneously, the radical polymerization of the different fluorinated acryl- $\mathrm{C}_{3} \mathrm{~F}_{6}$ and acryl- $\mathrm{C}_{7} \mathrm{~F}_{12}$ monomers occurs in situ to form polymeric structures that covalently attach to the phenylene layer. As a result, the quantitative grafting of fluorinated molecules in the form of a polymer film is covalently anchored to the $\mathrm{MoS}_{2}$ surface using a mild radical-based reaction. As a control reaction, a mixture of $\operatorname{MoS}_{2}$ flakes and vinyl monomers was prepared in the absence of the diazonium molecules, leading to the uncoated material. This control experiment confirms the dual role of the diazonium salt that, in the presence of $\mathrm{MoS}_{2}$ as reducing agent takes up an electron leading to aryl radicals ${ }^{32}$ that i) link to the $\mathrm{MoS}_{2}$ basal-plane to form a phenylene layer and ii) initiate the radical polymerization of the vinylic monomers in solution. After centrifugation and thoroughly washing off the black solids 
obtained, the functionalized $\mathrm{MoS}_{2}$, hereafter $\mathrm{MoS}_{2} @ \mathrm{C}_{3} \mathrm{~F}_{6}$ and $\mathrm{MoS}_{2} @ \mathrm{C}_{7} \mathrm{~F}_{12}$, were obtained and characterized.
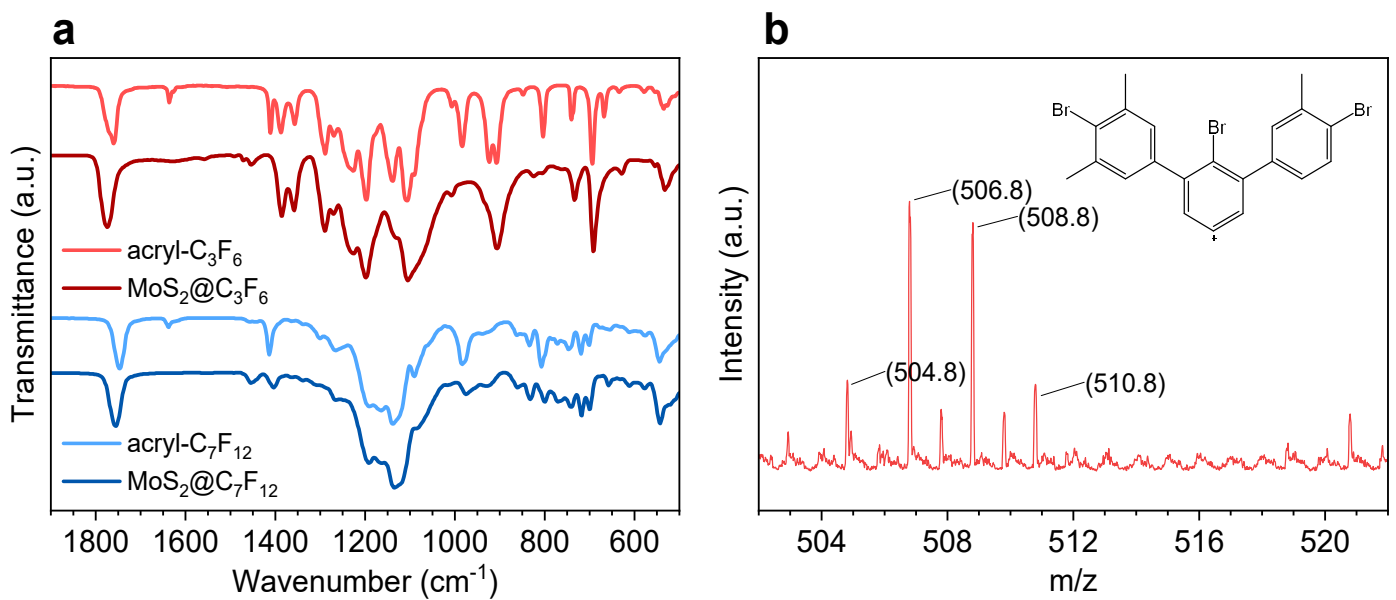

C

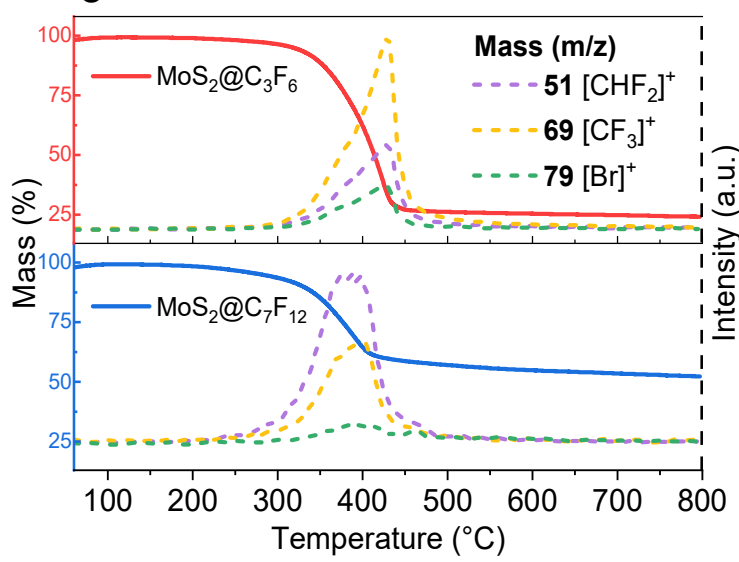

d

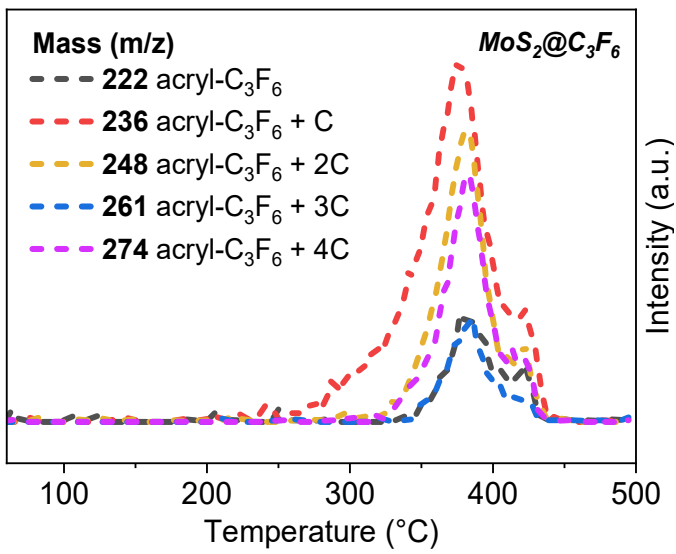

Figure 2. a) Infrared spectra of functionalized $M_{0} S_{2} @ C_{3} F_{6}$ (dark red) and $M_{0} S_{2} @ C_{7} F_{12}$ (dark blue) materials as compared to corresponding commercial fluorinated acryl monomers (light colours). b) Isotopic distribution of the base peak of MALDI-TOF measurements attributed to three covalently bonded bromoaryl molecules. c) Thermal profiles of functionalized $\mathrm{MoS}_{2} @ \mathrm{C}_{3} \mathrm{~F}_{6}$ (red) and $\mathrm{MoS}_{2} @ \mathrm{C}_{7} \mathrm{~F}_{12}$ (blue) materials with the corresponding coupled mass selected peaks detected upon thermal treatment as deduced from TGA-MS spectrometry. Molecular moieties detached correspond to $\mathrm{CHF}_{2}$ (purple), $\mathrm{CF}_{3}$ (yellow), and $\mathrm{Br}$ (green). d) In-depth analysis of the mass fragments detached upon thermal treatment in the $\mathrm{MoS}_{2} @ \mathrm{C}_{3} \mathrm{~F}_{6}$ coated material. The selected molecular fragments correspond to successive carbon additions and match with a vinylic polymer formation.

$\mathrm{MoS}_{2}$ polymer functionalization was evidenced by the feasible dispersibility and colloidal stability of $\mathrm{MoS}_{2} @ \mathrm{C}_{3} \mathrm{~F}_{6}$ and $\mathrm{MoS}_{2} @ \mathrm{C}_{7} \mathrm{~F}_{12}$ in organic solvents (i.e., acetonitrile and dichloromethane), which contrasts with the colloidal instability of $\mathrm{MoS}_{2}$ under similar conditions. We first analyzed the functionalization by infrared spectroscopy. Figure 2a depicts the ATR-FTIR spectra of the corresponding functionalized $\mathrm{MoS}_{2} @ \mathrm{C}_{3} \mathrm{~F}_{6}$ and $\mathrm{MoS}_{2} @ \mathrm{C}_{7} \mathrm{~F}_{12}$ 
materials compared to the commercial fluorinated molecules. The two pairs of spectra present similar bands arising from the fluorinated molecules, with particularly relevant differences. In the functionalized $\mathrm{MoS}_{2}$, the band at $1635 \mathrm{~cm}^{-1}$ attributed to the acryl vibration $(\vee \mathrm{C}=\mathrm{C}$ ) disappears, ${ }^{33}$ in agreement with a grafting reaction. This loss of the $C=C$ functionality is also confirmed by a shifting of the intense band at $\approx 1740 \mathrm{~cm}^{-1}$ attributed to the $\mathrm{C}=0$ vibration towards higher energies, consistent with the transformation of an $\alpha, \beta$-unsaturated ketone to a saturated one (Figure S5), as well as the loss of the band at $\approx 1410 \mathrm{~cm}^{-1}$, assigned to the $\mathrm{C}-\mathrm{H}$ vibration in $\mathrm{C}=\mathrm{C} .{ }^{34}$ Besides, the absence of the band at $2286 \mathrm{~cm}^{-1}$ attributed to the $\mathrm{N}-\mathrm{N}$ vibration of the $\mathrm{N}_{2}{ }^{+}$group in the diazonium salt ${ }^{35-37}$ confirms its elimination (Figure S6). The effect of the polymer grafting on $\mathrm{CE}-\mathrm{MoS}_{2}$ was then studied by Raman spectroscopy, where the characteristic J peaks $\left(154,226\right.$, and $\left.330 \mathrm{~cm}^{-1}\right)$ of CE-MoS 2 disappear upon functionalization (Figure S7).

Thermogravimetric analysis of the functionalized $\mathrm{MoS}_{2} @ \mathrm{C}_{3} \mathrm{~F}_{6}$ and $\mathrm{MoS}_{2} @ \mathrm{C}_{7} \mathrm{~F}_{12}$ as compared to the $\mathrm{CE}-\mathrm{MoS}_{2}$ were analyzed to estimate the degree of coverage. Profiles depicted in Figure 2c for the functionalized $\mathrm{MoS}_{2} @ \mathrm{C}_{3} \mathrm{~F}_{6}$ and $\mathrm{MoS}_{2} @ \mathrm{C}_{7} \mathrm{~F}_{12}$ show no significant mass loss below $300{ }^{\circ} \mathrm{C}$, which is followed by a weight loss in the temperature range $300-500{ }^{\circ} \mathrm{C}$ equivalent to $76 \%$ and 48\%, respectively for $\mathrm{MoS}_{2} @ \mathrm{C}_{3} \mathrm{~F}_{6}$ and $\mathrm{MoS}_{2} @ \mathrm{C}_{7} \mathrm{~F}_{12}$. These profiles contrast with the typically large thermal stability associated with $\mathrm{CE}-\mathrm{MoS}_{2}$ (see Figure S3) and should be attributed to the thermal decomposition of the grafted organic coating. Remarkably, the obtained large mass loss corresponding to a significant presence of organic coating contrasts with the typically moderate organic content obtained in truly molecular surface functionalization previously reported. ${ }^{19,21,38}$ This evidence supports the formation of a branched-like polymer film in $\mathrm{MoS}_{2} @ \mathrm{C}_{3} \mathrm{~F}_{6}$ and $\mathrm{MoS}_{2} @ \mathrm{C}_{7} \mathrm{~F}_{12}$ materials as illustrated in Figure 1. In an attempt to elucidate the moieties thermally detached from the functional hybrid coated material, thermogravimetric analysis was coupled to mass spectrometry (TGA-MS) (Figure $2 \mathrm{c}-\mathrm{d}$ ). Three main mass peaks with $\mathrm{m} / \mathrm{z}=51$, 69 , and 79 respectively attributed to $\mathrm{CF}_{2}$ and $\mathrm{CF}_{3}$ groups and a $\mathrm{Br}$ moiety were detected at 430 and $400{ }^{\circ} \mathrm{C}$, respectively for $\mathrm{MoS}_{2} @ \mathrm{C}_{3} \mathrm{~F}_{6}$ and $\mathrm{MoS}_{2} @ \mathrm{C}_{7} \mathrm{~F}_{12}$ materials (Figure 2b), which confirms the presence of fluorinated monomers and bromoaryl molecules in the coating shell. A more detailed analysis in the $\mathrm{MoS}_{2} @ \mathrm{C}_{3} \mathrm{~F}_{6}$ material evidence mass losses peaks with larger $\mathrm{m} / \mathrm{z}$ values, mainly $\mathrm{m} / \mathrm{z}=222,236,248,261$, and 274 (Figure $2 \mathrm{~d}$ ), which are attributed to successive additions of one carbon-chain fragment to a vinyl monomer (see Figure $\mathrm{S} 8$ for further details). These detected molecular fragments support the formation of the covalent coating via an induced radical polymerization of the acrylic monomers to form a fluorinated polymeric shell. 
MALDI-TOF spectrometry was then used to gain insight into the surface functionalization mechanism (Figure $2 b$ and S9). It was found that the base peak of the spectrum appears at $\mathrm{m} / \mathrm{z}$ $=(505,507,509,511)$, which is assigned to a molecular fragment comprising three covalently bonded bromoaryl molecules, as deduced from isotopic distribution. The detection of this large molecular fragment is in agreement with the further aryl radical attack to pre-grafted aryl species, supporting that the diazonium reaction proceeds by a radical mechanism. As a result, a polyphenylene sub-layer is directly anchored onto the $\mathrm{MoS}_{2}$ basal plane, acting as a covalent bridge between the $\mathrm{MoS}_{2}$ and the polymeric fluorinated coating, as previously reported. ${ }^{26,28-30}$
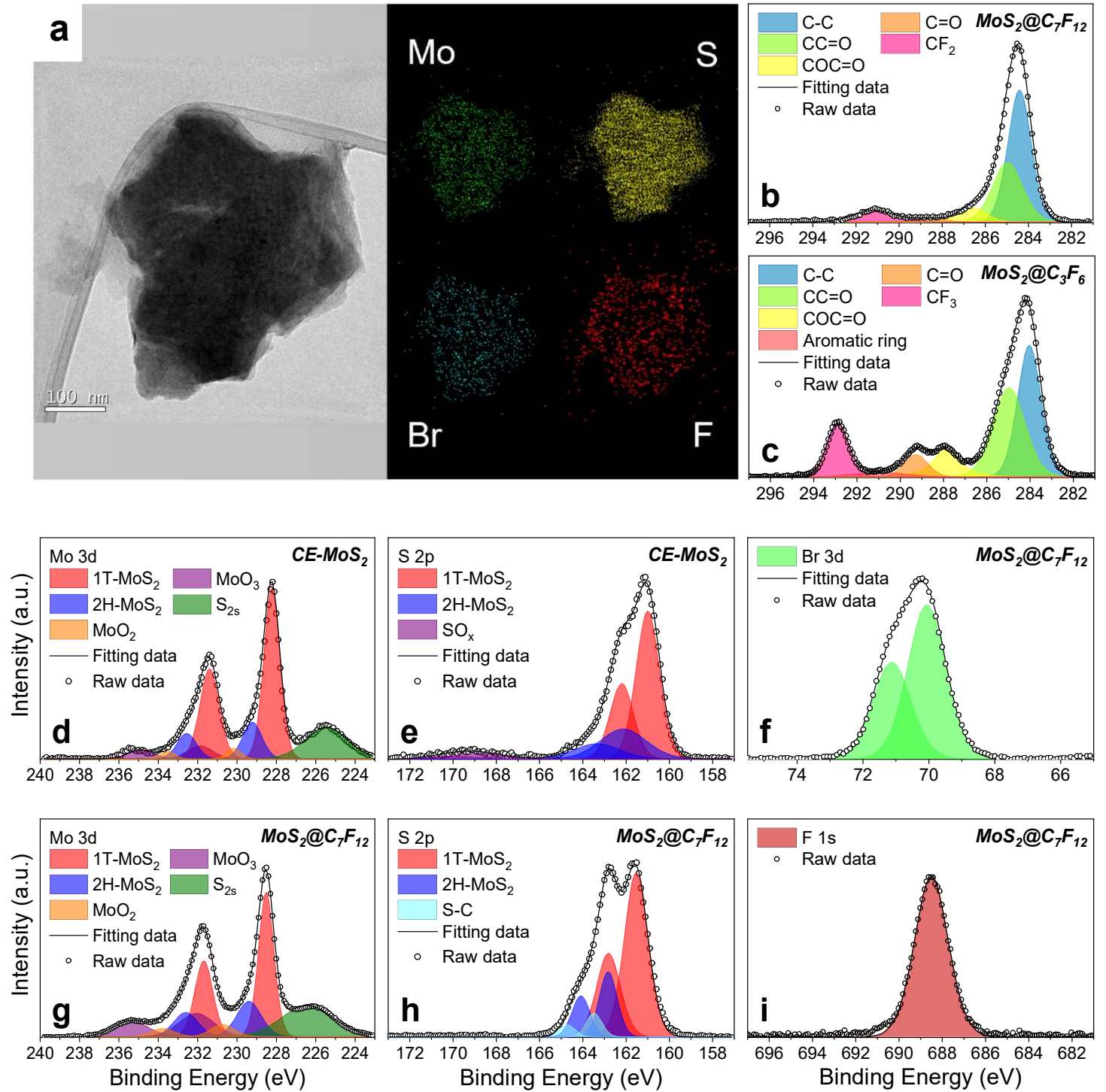

Figure 3. (Top) HR-TEM images of a flake of $\mathrm{MoS}_{2}$ decorated with the grafted molecules and a mapping that confirms the presence of $\mathrm{Br}$ and $\mathrm{F}$ (blue and red). (Bottom) XPS measurements of $\mathrm{CE}-\mathrm{MoS}_{2}$ and functionalized $\mathrm{MoS}_{2} @ \mathrm{C}_{7} \mathrm{~F}_{12}$ and $\mathrm{MoS}_{2} @ \mathrm{C}_{7} \mathrm{~F}_{12}$. 
The morphology of the functionalized $\mathrm{MoS}_{2} @ \mathrm{C}_{3} \mathrm{~F}_{6}$ and $\mathrm{MoS}_{2} @ \mathrm{C}_{7} \mathrm{~F}_{12}$ flakes was studied by means of HR-TEM. As Figure 3a shows, vertically aligned stages are formed after the functionalization. This is due to the merging of adjacent flakes as a result of the attraction between the carbon chains at the 2D interphase. The chemical composition of the coated flakes was studied by energy-dispersive $\mathrm{X}$-ray (EDX) analysis, revealing the presence of the main functional groups, represented by bromine (from the diazonium molecule) and fluorine (from the acryl molecules). The distribution of these elements along the flake is homogeneous, indicating the formation of a uniform coating.

The electronic signature and the composition of the $\mathrm{MoS}_{2} @ \mathrm{C}_{3} \mathrm{~F}_{6}$ and $\mathrm{MoS}_{2} @ \mathrm{C}_{7} \mathrm{~F}_{12}$ coated flakes were studied by X-ray Photoelectron spectroscopy (XPS). Given the comparable results obtained for the two materials, only the composite $\mathrm{MoS}_{2} @ \mathrm{C}_{7} \mathrm{~F}_{12}$ is discussed below (Figure 3b-i), whereas details for $\mathrm{MoS}_{2} @ \mathrm{C}_{3} \mathrm{~F}_{6}$ XPS spectra are described in the Supporting Material (Figure S10). Essentially, clear differences were observed between the polymer-coated and the control CE$\mathrm{MoS}_{2}$ materials. It is important to remark that $\mathrm{MoS}_{2}$ can present two main polytypes, i.e.: a hexagonal $2 \mathrm{H}$ phase and the tetragonal $1 \mathrm{~T}$ phase. The former phase is more abundant in $\mathrm{MoS}_{2}$ bulk crystals or mechanically exfoliated layers, whereas the latter is commonly obtained after chemically exfoliation processes, as in the present study. Focusing on the Mo 3d XPS spectra, the predominance of the $1 \mathrm{~T}$ phase can be discerned in $\mathrm{CE}-\mathrm{MoS}_{2}$, with peaks at $\approx 228.2 \mathrm{eV}$ for Mo $3 d_{5 / 2}$ and $\approx 231.4 \mathrm{eV}$ for Mo $3 d_{3 / 2}$ (Figure $3 d$ ). After functionalization with acryl- $C_{7} F_{12}$, the same $1 \mathrm{~T}$ phase predominance is maintained with peaks moving to $\approx 228.5$ and $\approx 231.7 \mathrm{eV}$, respectively for Mo $3 d_{5 / 2}$ and Mo $3 d_{3 / 2}$ (Figure $3 g$ ). Such blue-shifting is likely related to the loss of electron density at the CE-MoS interphase, produced by the electron transfer between the electronrich metallic $1 \mathrm{~T}-\mathrm{MoS}_{2}$ and the bromobenzene diazonium salt to form the aryl radicals. This phenomenon is also evidenced in the $S 2 p$ spectrum (Figure $3 e$, and $h$ ), which is moreover accompanied by the appearance of a brand-new doublet with $S 2 p_{3 / 2}$ at $\approx 163.5 \mathrm{eV}$ and $S 2 p_{1 / 2}$ $\approx 164.8 \mathrm{eV}$. This doublet is typically assigned to the presence of S-C bonds, ${ }^{16-18,39,40}$ suggesting a covalent functionalization of $\mathrm{MoS}_{2}$, which corresponds to a 7\% based on sulfur in the case of $\mathrm{MoS}_{2} @ \mathrm{C}_{7} \mathrm{~F}_{12}$ and 5\% for $\mathrm{MoS}_{2} @ \mathrm{C}_{3} \mathrm{~F}_{6}$, as expected for typical diazonium-based reactions. ${ }^{17}$ The presence of new bands in the carbon region is in good agreement with the anchored vinyl polymer, including the major groups $\mathrm{CF}_{2}$ and $\mathrm{CF}_{3}$ for $\mathrm{MoS}_{2} @ \mathrm{C}_{7} \mathrm{~F}_{12}$ and $\mathrm{MoS}_{2} @ \mathrm{C}_{3} \mathrm{~F}_{6}$, respectively (Figure $3 \mathrm{~b}-\mathrm{c}$ ). ${ }^{41}$ In addition, the presence of the new $\mathrm{F}$ 1s band at $688.5 \mathrm{eV}$ in the coated $\mathrm{MoS}_{2} @ \mathrm{C}_{7} \mathrm{~F}_{12}$ and $\mathrm{MoS}_{2} @ \mathrm{C}_{3} \mathrm{~F}_{6}$ endorse the existence of $\mathrm{CF}_{2}$ and $\mathrm{CF}_{3}$ groups from the fluorinated monomers (Figure 3i). ${ }^{41}$ Finally, in the $\mathrm{Br} 3 \mathrm{~d}$ XPS region, the characteristic $\mathrm{Br} 3 \mathrm{~d}$ doublet with the 
main peak at $70.0 \mathrm{eV}\left(\mathrm{Br}_{3} \mathrm{~d}_{5 / 2}\right)$ accompanied by a $1.10 \mathrm{eV}$ spin-orbit coupling can be seen, confirming the presence of bromobenzene in the coated materials (Figure $3 f$ ).

Among the different functionalities that can be incorporated into the $\mathrm{CE}-\mathrm{MoS}_{2}$ following this flexible vinylic polymerization reaction, hydrophobicity was first selected to clearly evidence the coating performance. Broadly, imparting surface hydrophobicity can be a key aspect of a $2 \mathrm{D}$ material system, which may prevent chemical instability, govern cell proliferation, improve antibacterial effects, or provide oil-water separation, among other characteristics. ${ }^{42-46}$ Coating performance was evaluated through contact angle measurements performed on pellets of the corresponding coated $\mathrm{MoS}_{2} @ \mathrm{C}_{3} \mathrm{~F}_{6}$ and $\mathrm{MoS}_{2} @ \mathrm{C}_{7} \mathrm{~F}_{12}$ materials as compared to the control CE$\mathrm{MoS}_{2}$ flakes (see Figure 4). Contact angle values of 110 and $150^{\circ}$ were obtained respectively for the coated $\mathrm{MoS}_{2} @ \mathrm{C}_{3} \mathrm{~F}_{6}$ and $\mathrm{MoS}_{2} @ \mathrm{C}_{7} \mathrm{~F}_{12}$ materials (Figure 4a and b, respectively), while in the case of the pristine CE-MoS 2 the water drops completely spread over the surface (Figure 4c). These results evidence that $\mathrm{MoS}_{2}$ truly becomes highly hydrophobic when coated through in situ formation of fluorinated polymers. The larger angles obtained in the $\mathrm{MoS}_{2} @ \mathrm{C}_{7} \mathrm{~F}_{12}$ material suggest that hydrophobicity is drastically affected by the number of $F$ atoms of the acryl molecules, resulting more hydrophobic when larger fluorinated acryl monomers are used. To evaluate this different hydrophobicity, deeper analysis of the polymerization will be required, as it is likely linked to the extent of dendritic structures formation.

a

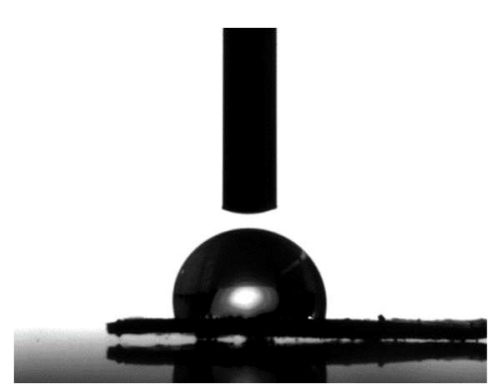

$\mathrm{MoS}_{2} @ \mathrm{C}_{3} \mathrm{~F}_{6}$

Max. angle: $110^{\circ}$ b

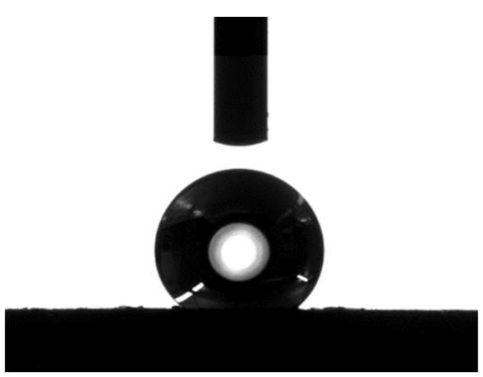

$\mathrm{MoS}_{2} @ \mathrm{C}_{7} \mathrm{~F}_{12}$

Max. angle: $150^{\circ}$
C

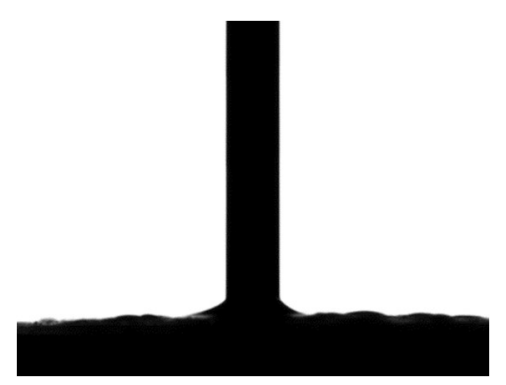

$\mathrm{CE}-\mathrm{MoS}_{2}$

Figure 4. Images of water drops in contact with the surface of $C E-M_{0} S_{2}, M_{0} S_{2} @ C_{3} F_{6}$, and $M_{0} S_{2} @ C_{7} F_{12}$ and the different contact angles that they exhibit.

\section{Conclusions}

We have successfully achieved the covalent adhesion of functional polymeric coatings onto CE$\mathrm{MoS}_{2}$ flakes in a synthesis occurring under mild conditions. The reaction uses the diazonium 
chemical reduction upon electron transfer from the metallic $1 \mathrm{~T}-\mathrm{MoS}_{2}$ to anchor a first phenylene layer, which acts as the base for the radical growth of branched vinyl polymers formed in situ. In this work, functional acryl monomers comprising hydrophobic groups and varying their chain lengths have been selected. In both cases, the covalent coating occurred in a fast and simple reaction and has been evidenced by multiple experimental techniques including TGA-MS and XPS. The coated materials exhibit large hydrophobic behaviour arising from the anchored fluorinated molecules of the organic coating, as evidenced by contact angle measurements. We anticipate that the flexibility of the reported covalent chemical functionalization will allow coating with practically any acrylate monomer to form functional polymeric coatings. The resulting strong interfacial bonding between the organic functional matrix and the $\mathrm{MoS}_{2}$ significantly expands the possibilities of the 2D material for numerous applications.

\section{Acknowledgements}

This work has been supported by the Spanish MICINN (Grant MAT2017-89993-R and Excellence Unit María de Maeztu MDM-2015-0538), the Generalitat Valenciana (SEJI2020/036), and from "la Caixa" Foundation (LCF/BO/PI19/11690022). Spanish MINECO is also acknowledged for predoctoral fellowships (to I.G.-M., R.T.-C., and M.M.-G) and a Ramón y Cajal Contract (RYC2019-027902-1).

\section{References}

1 M. Chhowalla, H. S. Shin, G. Eda, L. J. Li, K. P. Loh and H. Zhang, Nat. Chem., 2013, 5, 263275.

2 A. Eftekhari, J. Mater. Chem. A, 2017, 5, 18299-18325.

3 R. J. Toh, Z. Sofer and M. Pumera, J. Mater. Chem. A, 2016, 4, 18322-18334.

4 M. Samadi, N. Sarikhani, M. Zirak, H. Zhang, H. L. Zhang and A. Z. Moshfegh, Nanoscale Horiz., 2018, 3, 90-204.

5 J. Yang, A. Wang, S. Zhang, J. Liu, Z. Zhong and L. Chen, Phys. Chem. Chem. Phys., 2019, 21, 132-136.

6 J. Luxa, O. Jankovský, D. Sedmidubský, R. Medlín, M. Maryško, M. Pumera and Z. Sofer, 
Nanoscale, 2016, 8, 1960-1967.

P. Avouris, T. F. Heinz and T. Low, 2D materials: Properties and devices, Cambridge University Press, 2017.

A. Chaves, J. G. Azadani, H. Alsalman, D. R. da Costa, R. Frisenda, A. J. Chaves, S. H. Song, Y. D. Kim, D. He, J. Zhou, A. Castellanos-Gomez, F. M. Peeters, Z. Liu, C. L. Hinkle, S. H. Oh, P. D. Ye, S. J. Koester, Y. H. Lee, P. Avouris, X. Wang and T. Low, npj 2D Mater App, 2020, 4, 29.

$9 \quad$ M. A. Py and R. R. Haering, Can. J. Phys., 1983, 61, 76-84.

10

C. Tan, X. Cao, X. J. Wu, Q. He, J. Yang, X. Zhang, J. Chen, W. Zhao, S. Han, G. H. Nam, M. Sindoro and H. Zhang, Chem. Rev., 2017, 117, 6225-6331.

11 Q. Tang and D. E. Jiang, Chem. Mater., 2015, 27, 3743-3748.

12 M. Morant-Giner, R. Sanchis-Gual, J. Romero, A. Alberola, L. García-Cruz, S. Agouram, M. Galbiati, N. M. Padial, J. C. Waerenborgh, C. Martí-Gastaldo, S. Tatay, A. Forment-Aliaga and E. Coronado, Adv. Funct. Mater., 2018, 28, 1706125.

L. Daukiya, J. Seibel and S. De Feyter, Adv. Phys. X, 2019, 4, 1625723.

S. Bertolazzi, M. Gobbi, Y. Zhao, C. Backes and P. Samorì, Chem. Soc. Rev., 2018, 47, 68456888.

15 A. Hirsch and F. Hauke, Angew. Chem. Int. Ed., 2018, 57, 4338-4354.

16 R. Quirós-Ovies, M. Vázquez Sulleiro, M. Vera-Hidalgo, J. Prieto, I. J. Gómez, V. Sebastián, J. Santamaría and E. M. Pérez, Chem. Eur. J., 2020, 26, 6629-6634.

17 K. C. Knirsch, N. C. Berner, H. C. Nerl, C. S. Cucinotta, Z. Gholamvand, N. McEvoy, Z. Wang, I. Abramovic, P. Vecera, M. Halik, S. Sanvito, G. S. Duesberg, V. Nicolosi, F. Hauke, A. Hirsch, J. N. Coleman and C. Backes, ACS Nano, 2015, 9, 6018-6030.

18 D. Voiry, A. Goswami, R. Kappera, C. D. C. C. E. Silva, D. Kaplan, T. Fujita, M. Chen, T. Asefa and M. Chhowalla, Nat. Chem., 2015, 7, 45-49.

19 E. Er, H. L. Hou, A. Criado, J. Langer, M. Möller, N. Erk, L. M. Liz-Marzán and M. Prato, Chem. Mater., 2019, 31, 5725-5734.

20 L. Zhou, B. He, Y. Yang and Y. He, RSC Adv., 2014, 4, 32570-32578.

21 P. Vishnoi, A. Sampath, U. V. Waghmare and C. N. R. Rao, Chem. Eur. J., 2017, 23, 886- 
895.

22 L. Daukiya, J. Teyssandier, S. Eyley, S. El Kazzi, M. C. Rodríguez González, B. Pradhan, W. Thielemans, J. Hofkens and S. De Feyter, Nanoscale, 2021, 13, 2972-2981.

L. Assies, C. Fu, P. Kovaříček, Z. Bastl, K. A. Drogowska, J. Lang, V. L. P. Guerra, P. Samorì,

E. Orgiu, D. F. Perepichka and M. Kalbáč, J. Mater. Chem. C, 2019, 7, 12240-12247.

H. Cheng, N. Dong, T. Bai, Y. Song, J. Wang, Y. Qin, B. Zhang and Y. Chen, Chem. Eur. J., 2016, 22, 4500-4507.

V. Mévellec, S. Roussel, L. Tessier, J. Chancolon, M. Mayne-L'Hermite, G. Deniau, P. Viel and S. Palacin, Chem. Mater., 2007, 19, 6323-6330.

A. Gohier, F. Nekelson, M. Helezen, P. Jegou, G. Deniau, S. Palacin and M. MayneL'Hermite, J. Mater. Chem., 2011, 21, 4615-4622.

27 D. J. Eyckens, C. L. Arnold, J. D. Randall, F. Stojcevski, A. Hendlmeier, M. K. Stanfield, J. Pinson, T. R. Gengenbach, R. Alexander, L. C. Soulsby, P. S. Francis and L. C. Henderson, ACS Appl. Mater. Interfaces, 2019, 11, 41617-41625.

28 A. Mesnage, M. A. Magied, P. Simon, N. Herlin-Boime, P. Jégou, G. Deniau and S. Palacin, J. Mater. Sci., 2011, 46, 6332-6338.

M. Giménez-Marqués, E. Bellido, T. Berthelot, T. Simón-Yarza, T. Hidalgo, R. SimónVázquez, Á. González-Fernández, J. Avila, M. C. Asensio, R. Gref, P. Couvreur, C. Serre and P. Horcajada, Small, 2018, 14, 1801900.

30 M. Benzaqui, R. Semino, F. Carn, S. R. Tavares, N. Menguy, M. Giménez-Marqués, E. Bellido, P. Horcajada, T. Berthelot, A. I. Kuzminova, M. E. Dmitrenko, A. V. Penkova, D. Roizard, C. Serre, G. Maurin and N. Steunou, ACS Sustain. Chem. Eng., 2019, 7, 66296639.

31 J. Greenwood, T. H. Phan, Y. Fujita, Z. Li, O. Ivasenko, W. Vanderlinden, H. Van Gorp, W. Frederickx, G. Lu, K. Tahara, Y. Tobe, H. Uji-I, S. F. L. Mertens and S. De Feyter, ACS Nano, $2015,9,5520-5535$.

32 C. Galli, Chem. Rev., 1988, 88, 765-792.

33 P. Carmona and J. Moreno, J. Mol. Struct., 1982, 82, 177-185.

34 S. Roberge and M. A. Dubé, J. Appl. Polym. Sci., 2016, 133, 43574. 

Chem. Eur. J., 2015, 21, 15583-15588.

39 M. Vera-Hidalgo, E. Giovanelli, C. Navío and E. M. Pérez, J. Am. Chem. Soc., 2019, 141, 3767-3771.

40 M. Cai, F. Zhang, C. Zhang, C. Lu, Y. He, Y. Qu, H. Tian, X. Feng and X. Zhuang, J. Mater. Chem. A, 2018, 6, 138-144.

41 C. M. Kassis, J. K. Steehler, D. E. Betts, Z. Guan, T. J. Romack, J. M. DeSimone and R. W. Linton, Macromolecules, 1996, 29, 3247-3254.

42 A. Vilčnik, I. Jerman, A. Š. Vuk, M. Koželj, B. Orel, B. Tomšič, B. Simončič and J. Kovač, Langmuir, 2009, 25, 5869-5880.

43 X. Zhang, Z. Li, K. Liu and L. Jiang, Adv. Funct. Mater., 2013, 23, 2881-2886.

44 N. Han, Z. Zhang, H. Gao, Y. Qian, L. Tan, C. Yang, H. Zhang, Z. Cui, W. Li and X. Zhang, ACS Appl. Mater. Interfaces, 2020, 12, 2926-2934.

45 Q. Sun, B. Aguila, J. A. Perman, T. Butts, F. S. Xiao and S. Ma, Chem, 2018, 4, 1726-1739.

46 T. Yu, D. Mathias, S. Lu, W. Xu, M. Naushad, S. Szunerits and R. Boukherroub, Sep. Purif. Technol., 2020, 238, 116420. 